\title{
Evolution saisonnière des niveaux plasmatiques de thy- roxine et de triiodothyronine chez le taureau post-pubère en France et en Irak. Variations concomitantes des taux de $\mathrm{LH}$ et de testostérone
}

\author{
A. Shubbur, M. Goffaux et M. Thibier \\ Laboratoire pour le contrôle des reproducteurs - UNCEIA - 13, rue Joüet - B.P. 65 \\ 94703 Maisons-Alfort, France
}

(reçu le 29 septembre 1988, accepté le 11 mai 1989)

Résumé - Une enquête a été menée dans 2 stations de contrôle zootechnique individuel situées l'une en France (A), l'autre en Irak (B). Dans les deux stations, aux solstices et aux équinoxes, 30 taureaux Holstein de 15 mois ont fait l'objet de prélèvements de sang, en vue de la détermination des concentrations plasmatiques de $\mathrm{LH}$, testostérone, thyroxine et triiodothyronine.

Pour $\mathrm{LH}$, aucun effet saisonnier cohérent n'a été trouvé. Pour la testostérone, les valeurs maximales $(3,4 \mathrm{ng} / \mathrm{ml})$ ont été obtenues en décembre dans les deux stations. A la station $\mathrm{A}$, comme à la station $B$, la thyroxine passe en décembre par un sommet atteignant respectivement $64,6 \mathrm{ng} / \mathrm{ml}$ et $77,8 \mathrm{ng} / \mathrm{ml}$ et tombe en juin aux valeurs les plus basses, respectivement égales à $49,4 \mathrm{ng} / \mathrm{ml}$ et $65,6 \mathrm{ng} / \mathrm{ml}$. La différence est significative à la station $A(P<0,001)$. Pour T3, la chute de décembre $(1,42 \mathrm{ng} / \mathrm{ml}$ et $1,68 \mathrm{ng} / \mathrm{ml}$ respectivement en $A$ et $B)$ à juin $(1,09 \mathrm{ng} / \mathrm{ml}$ et $1,26 \mathrm{ng} / \mathrm{ml})$ a à peu près la même valeur relative et est également significative $(P<0,005)$ dans les deux stations.

L'effet défavorable des températures élevées sur la qualité de la semence ne paraît pas lié à une altération de la fonction thyroïdienne.

bovin — mâle — fonction sexuelle - thyroïde — variations saisonnières

Summary - Seasonal variations of blood plasma thyroxine and triiodothyronine in the postpubertal bull in France and Iraq. Concomitant variation of LH and testosterone. An inquiry was conducted in 2 performance testing stations, $A$ and $B$ located in France and Iraq, respectively. In both stations, at solstice and equinox, thirty 15 month-old Holstein bulls were blood sampled for plasma $\mathrm{LH}$, testosterone, thyroxine and triiodothyronine determination.

For $L H$, no coherent seasonal effect was found. As regards testosterone, maximal mean values were obtained in December in both stations $(3.4 \mathrm{ng} / \mathrm{ml})$. In $A$ as well as in $B$, thyroxine peaked in December reaching $64.6 \mathrm{ng} / \mathrm{ml}$ and $77.8 \mathrm{ng} / \mathrm{ml}$, respectively, and falling down to $49.4 \mathrm{ng} / \mathrm{ml}$ and $65.6 \mathrm{ng} / \mathrm{ml}$, respectively in June. The difference was significant for $A(P<0.001)$. For $T 3$, the fall from December (1.42 $\mathrm{ng} / \mathrm{ml}$ in $A$ and $1.68 \mathrm{ng} / \mathrm{ml}$ in $B$ ) to June $(1.09 \mathrm{ng} / \mathrm{ml}$ in $A$ and $1.26 \mathrm{ng} / \mathrm{ml}$ in $B$ ) resulted in about the same relative value and was significant $(P<0.005)$ in both stations.

The detrimental effect of high temperatures on semen quality does not seem to be mediated by an alternation of thyroid function.

Bull - sexual function - thyroid gland - seasonal variations 


\section{Introduction}

Les altérations du sperme de taureau sous l'effet du stress thermique ont été étudiées avec précision dans des étables climatisées (Stephan et al., 1971) mais souvent sans acclimatement préalable des animaux. Dans les pays tropicaux et subdésertiques, malgré certaines exceptions (Al-Haboby, 1977; Badawy, 1979), la saison chaude entraîne une baisse de la qualité du sperme (Misra et Sengupta, 1965; Igboeli et Rakha, 1971; Igboeli et al., 1987; Kumi-Diaka et al., 1981; Amir et al., 1982), parfois à peine perceptible, notamment chez les taureaux autochtones, parfois importante, avec une chute de plus de $25 \%$ du taux de spermatozoïdes mobiles ou une élévation de plus de $50 \%$ des anomalies morphologiques secondaires.

Or, à la différence de ce qu'on constate pour le bélier ou le bouc, chez le taureau les concentrations de LH et de testostérone ne sont pas affectées par des variations saisonnières unanimement reconnues (Thibier, 1975a, 1976; Downey et al., 1984; Parkinson, 1985). Quant aux températures élevées, appliquées expérimentalement, elles dépriment les niveaux de $\mathrm{LH}$ et de testostérone (Rhynes et Ewing, 1973; Minton et al., 1981), mais cet effet est moins net que celui qu'elles exercent sur la fonction thyroïdienne (Yousef et al., 1967; Magdub et al., 1982; Baccari et al., 1983).

Pour déterminer s'il existe une base endocrinienne à la moindre qualité du sperme récolté au cours de l'été en Irak, une étude des variations saisonnières des niveaux hormonaux a été menée dans 2 stations d'élevage de taurillons futurs reproducteurs, l'une en lle-de-
France, l'autre proche de Bagdad. Comme l'étude concernait la fonction sexuelle, nous l'avons orientée vers les dosages de $\mathrm{LH}$ et de testostérone, auxquels on a ajouté ceux des hormones thyroüdiennes du fait de l'importance des variations thermiques en Irak.

\section{Matériel et Méthodes}

La station A, située à $100 \mathrm{~km}$ au sud de Paris, a un climat tempéré. La température moyenne mensuelle passe de $3,0^{\circ} \mathrm{C}$ en janvier à $18,6^{\circ} \mathrm{C}$ en juillet. La latitude $\left(48^{\circ}\right.$ Nord) entraîne une variation de la photopériode de 8,2 à $15,8 \mathrm{~h}$. A la station irakienne de $\mathrm{B}$, par $33^{\circ}$ de latitude Nord, la photopériode varie de 9,8 à $14,2 \mathrm{~h}$, mais la température moyenne mensuelle passe de $10,7^{\circ} \mathrm{C}$ en janvier à $35,2^{\circ} \mathrm{C}$ en juillet, la moyenne des maxima dépassant $41^{\circ} \mathrm{C}$ de juin à août (contre $21,5^{\circ} \mathrm{C}$ à la station $\mathrm{A}$ ). Ces températures estivales s'accompagnent d'une très faible humidité relative ( $23 \%$ en juillet, contre $71 \%$ à la station A).

Dans chacune des stations, les animaux objet de l'enquête étaient des taureaux Holstein de 13 à 17 mois entretenus dans des conditions de stabulation et d'alimentation contrôlées et constantes au cours des années successives. A chaque solstice et à chaque équinoxe, 30 taureaux par station ont été l'objet d'un prélèvement de sang à la veine jugulaire en vue du dosage des hormones $\mathrm{LH}$, testostérone, thyroxine $\left(T_{4}\right)$ et triiodothyronine $\left(T_{3}\right)$. Les méthodes de dosage radio-immunologique employées pour $\mathrm{LH}$ et la testostérone ont été décrites par Thibier (1975a, 1976). Les concentrations de $T_{4}$ et $T_{3}$ ont été déterminées à l'aide de méthodes et d'un matériel disponibles dans le commerce 1 . L'étude a été menée sur 270 taureaux de décembre 1983 à décembre 1985 à la station A et sur 150 taureaux, de mars 1984 à mars 1985, à la station B.

Pour étudier l'effet de l'année à la station $A$ et comparer les 2 stations au cours de l'été et de l'hiver qui leur étaient communs, on a supprimé les données de décembre 1985 à la station A et celles de mars 1983 à la station $B$.

${ }_{1} \mathrm{~T}_{4} \mathrm{~K}$ et $\mathrm{T}_{3} \mathrm{~K}$ International CIS. Sensibilité pour $\mathrm{T}_{4}: 8 \mathrm{ng} / \mathrm{ml}$, pour $\mathrm{T}_{3}: 0,1 \mathrm{ng} / \mathrm{ml}$. Précision pour $T_{4}: 4 \%$ intra- et $7 \%$ interdosage, pour $T_{3}$ : environ $7 \%$ intra- et interdosage. 


\section{Résultats}

L'évolution des concentrations plasmatiques des différentes hormones selon la date de prélèvement est représentée par les graphiques des figures 1 et 2 .

Les concentrations moyennes générales de $\mathrm{LH}, \mathrm{T}, \mathrm{T}_{4}$ et $\mathrm{T}_{3}$ sont respectivement 2,$83 ; 2,65 ; 56,4$ et $1,28 \mathrm{ng} / \mathrm{ml}$ à la station $A$ et 3,$08 ; 2,68 ; 71,9$ et $1,42 \mathrm{ng} / \mathrm{ml}$ pour les mêmes hormones à la station $B$. Les niveaux moyens de $\mathrm{LH}, \mathrm{T}_{4}$ et $\mathrm{T}_{3}$ diffèrent dans les deux stations $(P<0,001)$. Pour $\mathrm{LH}$, l'effet de la date de prélèvement est significatif dans les 2 stations. II s'explique à $A$ par ceux du mois, de l'année et de l'interaction mois $x$ année $(P<0,001)$. Juin est plus élevé que décembre à la station $\mathrm{B}(P<0,001)$. Les variations de la testostérone entre les périodes de prélèvement sont plus nettes à la station $\mathrm{B}(P<0,001)$ qu'à la station $\mathrm{A}$ $(P<0,05)$.

Dans les 2 stations $A$ et $B$, la thyroxine atteint les valeurs les plus élevées en décembre : $64,6 \mathrm{ng} / \mathrm{ml} 2$ et $77,8 \mathrm{ng} / \mathrm{ml}$, et les plus faibles en juin : $49,4 \mathrm{ng} / \mathrm{ml} 3$ et $65,6 \mathrm{ng} / \mathrm{ml}$. Cette différence n'est significative $(P<0,001)$ qu'à la station $A$, où le mois, l'année et l'interaction mois $x$ année sont également significatifs $(P<0,001)$. Quant à l'évolution saisonnière de la triiodothyronine, elle est significative dans les 2 stations $(P<0,001)$, avec une interaction mois $\mathrm{x}$ année significative $(P<0,001)$ à la station $\mathrm{A}$. La chute significative de $\mathrm{T}_{3}$ $(P<0,005)$ entre décembre $(1,42 \mathrm{ng} / \mathrm{ml}$ à $A$ et $1,68 \mathrm{ng} / \mathrm{ml}$ à $B)$ et juin $(1,09 \mathrm{ng} / \mathrm{ml}$ à $A$ et $1,26 \mathrm{ng} / \mathrm{ml}$ à $B$ ) est, en valeur relative, à peu près la même dans les 2 stations.

\section{Discussion}

Pour $\mathrm{LH}$, le maximum de juin à la station A est assorti d'une forte interaction mois $x$ année précisément due à ce mois; Aussi ne peut-on pas lui accorder une signification générale. La supériorité de $B$ sur $A$, en juin 1984, ne peut s'expliquer ni par un effet de la photopériode, qui varie moins à $B$ qu'à $A$, ni par un effet de la température, lequel devrait être négatif, comme dans l'expérience de Minton et al. (1981).

Pour la testostérone, l'absence de variation saisonnière à la station $A$ s'accorde avec les observations faites en pays tempérés (Thibier, 1975b, 1976; Downey et al., 1984; Parkinson, 1985). Le maximum de décembre 1984 à la station $\mathrm{B}$ doit être relativisé compte tenu de la différence trouvée entre mars 1984 et mars 1985 dans cette station.

Pour la thyroxine, l'absence, dans cette étude, de liaison entre l'élévation de la température ambiante et la chute de concentration entre décembre et juin, s'accorde avec la conclusion résultant de la confrontation de 2 séries de données : celles de Pethes et al. (1979), qui observent chez des taureaux une baisse estivale profonde en Hongrie, et celles de Magdub et al. (1982), qui ne notent qu'une réduction de $16 \%$ après le passage de vaches en lactation à la température de $31,2^{\circ} \mathrm{C}$. Le fait que la chute estivale ne soit pas plus faible à la station $A$ qu'à la station $B$ ne s'explique pas par un effet de la photopériode, les hormones thyroïdiennes n'étant pas affectées chez le taureau par une modification de ce facteur (Tucker, 1982).

L'évolution de la triiodothyronine est voisine de celle de $\mathrm{T}_{4}$, ce qui semble

2 Moyenne des valeurs de 1983 et 1984 .

3 Moyenne des valeurs de 1984 et 1985. 
logique puisque $80 \%$ de la $\mathrm{T}_{3}$ produite dans l'organisme provient de la désiodation de $\mathrm{T}_{4}$ au niveau des tissus (De Visscher et Burger, 1980).

A la différence de l'évolution des concentrations plasmatiques de $\mathrm{LH}$ et de la testostérone, celle qu'on observe dans cette étude pour les hormones thyroï- diennes paraît correspondre à un effet saisonnier cohérent. Cependant, la mise dans un environnement chaud entraîne bien d'autres altérations de l'état général du bovin (Bond et McDowell, 1972; Stephan et Dycka, 1972; Magdub et al., 1982), y compris des variations du niveau du cortisol et la montée de celui des catécholamines (Alvarez et Johnson, 1973).
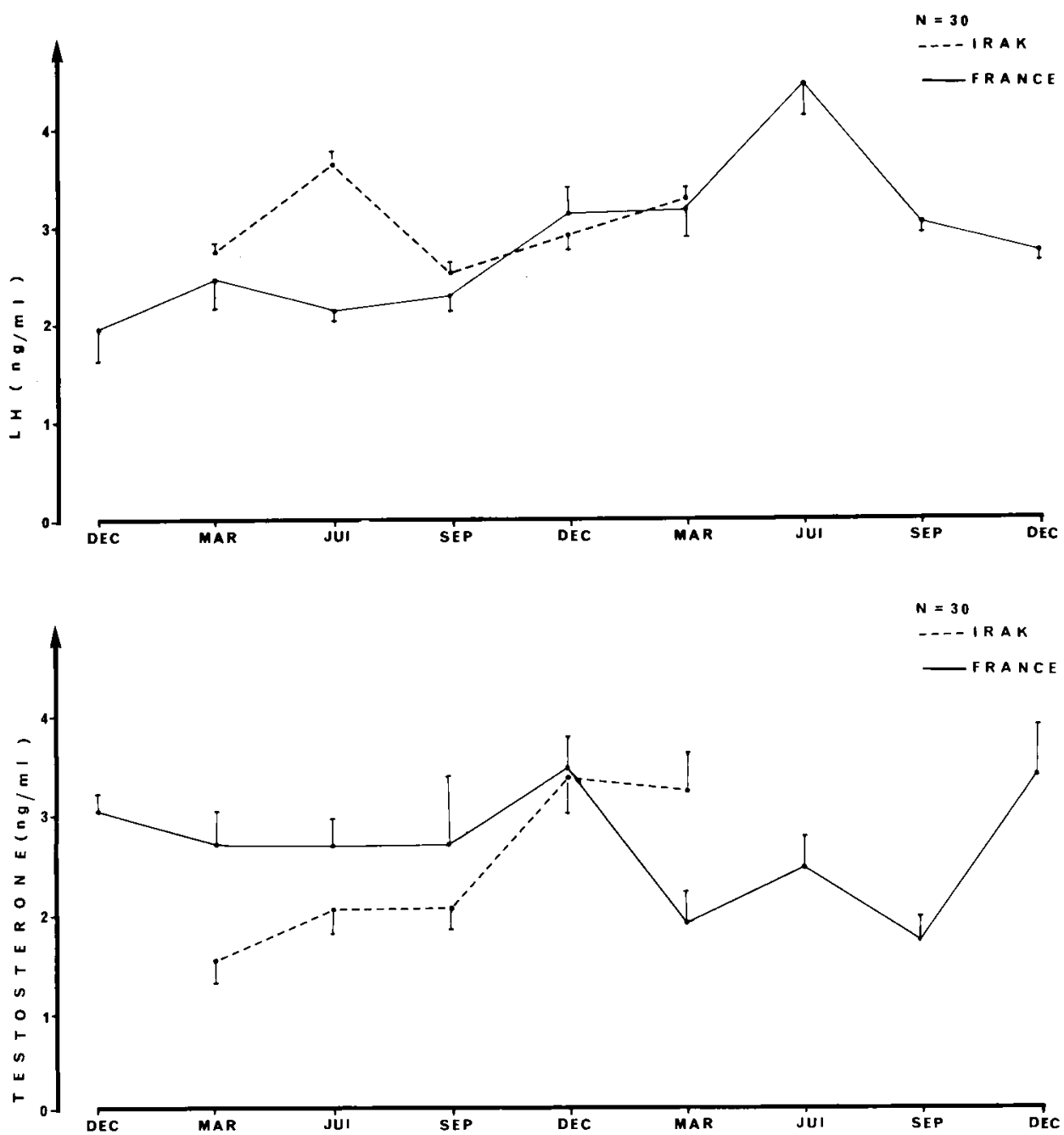

Fig. 1. Evolution des concentrations plasmatiques de LH et de testostérone. Moyennes assorties de leur écart type. $N=30$ taureaux par point. 
En outre, l'élévation de la température du testicule, dont on connaît les effets sur la spermatogenèse (Waites et Ortavant, 1968; Van Demark et Free, 1970), peut également se produire malgré les mécanismes naturels de défense (Waites, 1970). Associé à ces considérations, le maintien de niveaux élevés de $T_{4}$ et de $T_{3}$ à la station $B$ au cours de l'été, laisse assez peu de place à l'hypothèse d'une médiation thyroïdienne pour rendre compte de l'effet défavorable des températures élevées sur la qualité du sperme de taureau.
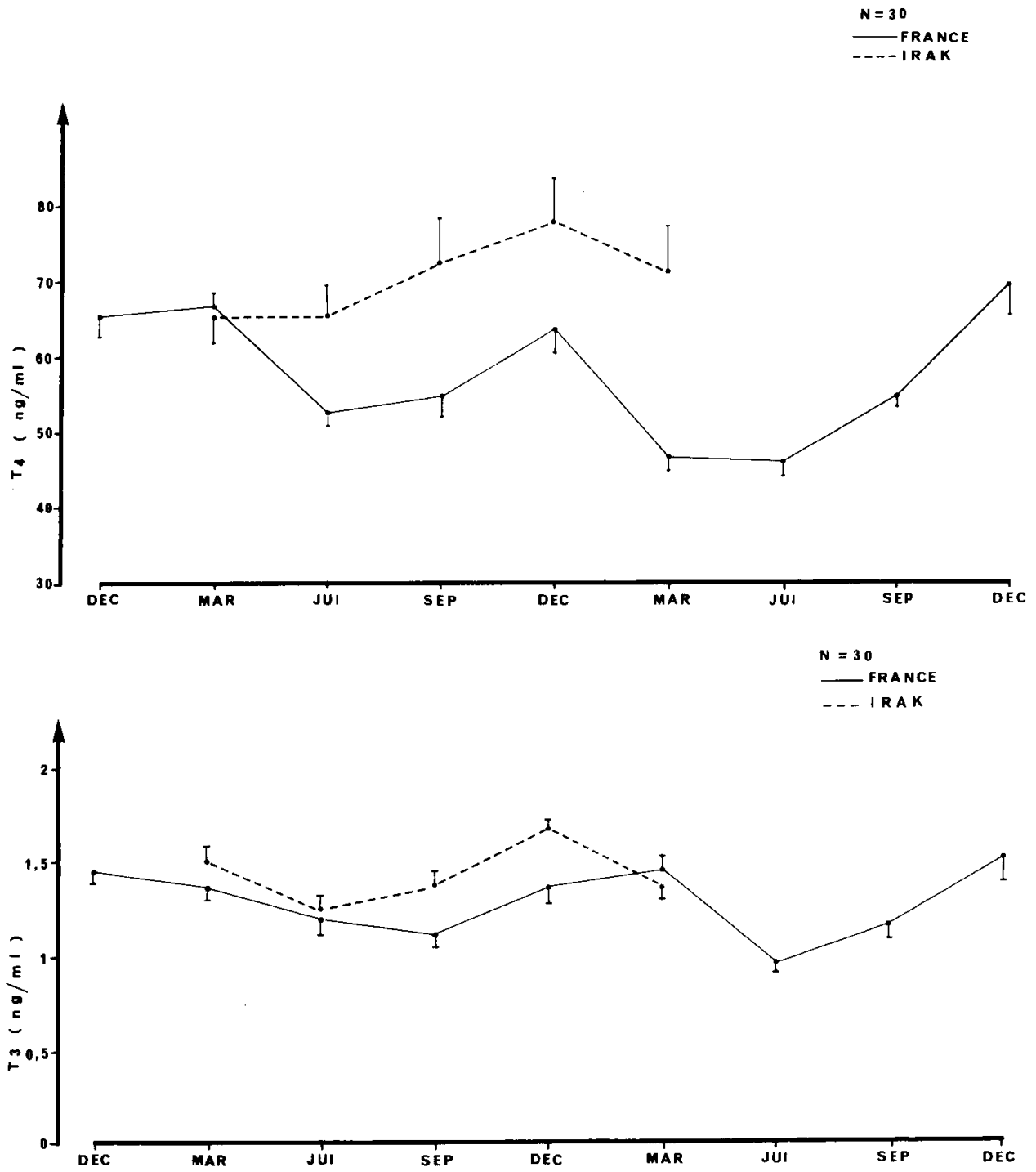

Fig. 2. Evolution des concentrations plasmatiques de thyroxine et de triiodothyronine. Moyennes assorties de leur écart type. $N=30$ taureaux par point. 


\section{Remerciements}

Les auteurs expriment leurs vifs remerciements aux responsables de la station de contrôle zootechnique de la CECNA à Amilly (Loiret) et du centre d'insémination artificielle d'Abou Grab (|rak).

\section{Références}

Alvarez M.B. \& Johnson H.D. (1973) Environmental heat exposure on cattle plasma catecholamines and glucocorticoïds. J. Dairy Sci. 56, 189-194

Amir D., Bar-El M., Kalay D. \& Schindler H. (1982) The contribution of bulls and cows to the seasonal differences in the fertility of dairy cattle in Israel. Anim. Reprod. Sci. 5, 93-98

Al-Haboby A. (1977) Studies on some semen characteristics of crossbred Friesian bulls and the effect of some diluents and dilution rates on spermatozoan motility during storage. Thesis. College of Agriculture, University of Bagdad, Iraq, p. 195

Baccari F. H. Jr., Johnson H.D. \& Leroy-Hahn G. (1983) Environmental heat effect on growth, plasma $\mathrm{T}_{3}$ and postheat compensatory effects on Holstein calves. Proc. Soc. Exp. Biol. Med. 173, 312-318

Badawy A.B.A. (1979) Seasonal variation in sexual desire and semen characters of Friesian bulls. Assiuf Vet. Med. J. 5, 287-299

Bond J. \& McDowell R.E. (1972) Reproductive performance and physiological responses of beef females as affected by a prolonged high environmental temperature. J. Anim. Sci. 35, 820-829

De Visscher M. \& Burger A. (1980) Evaluation of thyroid function : diagnostic procedures in thyroid diseases. In : The thyroid gland (De Visscher, ed.) Raven Press, New York, 169214

Downey B.R., Peirce A.R.J., \& Sanford L.M. (1984) Seasonal differences in pituitary and testicular function in bulls. 10th Int. Congr. on Animal Reproduction and A.I., Urbana, vol. II, 146

Igboeli G; \& Rakha A.M. (1971) Seasonal changes in the ejaculate characteristics of
Angoni (short horn zebu) bulls. J. Anim. Sci. 33, 651-654

Igboeli G., Nwakalor L.N., Orji B.I. \& Onvora G.I. (1987) Seasonal variation in the semen characteristics of Muturu (Bos brachyceros) bulls. Anim. Reprod. Sci. 14, 31-38

Kumi-Diaka J., Vagaratman V. \& Rwuaan J.S. (1981) Seasonal and age-related changes in semen quality and testicular morphology of bulls in a tropical environment. Vet. Rec. 108, 13-15

Magdub A., Johnson H.D. \& Belyea R.L. (1982) Effects of environmental heat and dietary fiber on thyroid physiology of lactating cows. $J$. Dairy Sci. 65, 2323-2331

Minton J.E., Wettemann R.P., Meyerhoeffer D.C., Intz R.L. \& Turman E.J. (1981) Serum luteinizing hormone and testosterone in bulls during exposure to elevated ambient temperature. J. Anim. Sci. 53, 1551-1557

Misra M.S. \& Sengupta B.P. (1965) Climatic environment and reproductive behaviour of buffaloes. III. Observations on housing conditions. Indian J. Dairy Sci. 18, 130-133

Parkinson T.J. (1985) Seasonal variation in semen quality of bulls and correlations with metabolic and endocrine parameters. Vet. Rec. 117, 303-307

Pethes G., Losonczy S. \& Rudas P. (1979) Interrelationship of serum thyroxine concentration with age and seasonal changes in growing bulls. Acta Vet. 29, 89-96

Rhynes W.E. \& Ewing L.L. (1973) Testicular endocrine function in Hereford bulls exposed to high ambient temperature. Endocrinology 92, 509-515

Stephan W.E., Lorrman W. \& Dycka J. (1971) Zur Problematik der Klimaempfindlichkeit bei Haustieren (111). Auswirkungen experimenteller Wärmebelastungen auf Bullen. 1. Mitteilung : Einige merkbare Eigenschaften des Ejakulates. Zuchthygiene 1, 19-38

Stephan W.E. \& Dycka J. (1972) Zur Problematik der Klimaempfindlichkeit bei Haustieren (VIII). Auswirkungen experimenteller Wärmebelastungen auf Bullen. 3. Mitteilung. Atmungsund Herztrequenz, Rektal-und Hauttemperatur, Wasseraufnahme sowie einige Blutmesswerte. Zuchthygiene 7, 71-82

Sundby A. \& Tollman R. (1978) Plasma testosterone in bulls. Seasonal variation. Acta Vet. Scand. 19, 263-268 
Thibier M. (1975a) Variations circadiennes de la LH plasmatique chez le jeune taurillon. Ann. Endocrinol. 36, 205-210

Thibier M. (1975b) Peripheral plasma testosterone concentrations in bulls around puberty. J. Reprod. Fert. 42, 567-569

Thibier M. (1976) Diurnal testosterone and 17 alpha hydroxyprogesterone in peripheral plasma of young post-pubertal bulls. Acta Endocrinol. 81, 623-634

Tucker A.H. (1982) Seasonality in cattle. Theriogenology 17, 53-59

Van Demark N.L. \& Free M.J. (1970) Temperature effects. In: The Testis, vol. III (Johnson
A.D., Gomes W.R. \& Van Dermark N.L., eds) Acad. Press, vol. III, 233-312

Waites G.M.H. (1970) Temperature regulation and the testis. In : The Testis (Johnson A.D., Gomes W.H. \& Van Dermark N.L., eds), vol. 1, 241-279

Waites G.M.H. \& Ortavant R. (1968) Effets précoces d'un $\gg$ brève élévation de la température testiculaire sur la spermatogenèse du bélier. Ann. Biol. Anim. Biochim. Biophys. 8, 323-331

Yousef M.K., Kibler H.H. \& Johnson H.D. (1967) Thyroid activity and heat production in cattle following sudden ambient temperature changes. J. Anim. Sci. 26, 142-148 